\title{
Case Report: Ultrasound Detection of IGG4 Related Disease Involving the Submandibular and Parotid Glands in Adolescent Age Group
}

\section{Rajesh C Kamble ${ }^{1 *}$, Alpana N Joshi ${ }^{2}$ and Pravin Mestry ${ }^{1}$}

${ }^{1}$ Radiodiagnosis, Shobha Diagnostic Centre, Dhiraj Apartments, Mumbai Malad East, India

${ }^{2}$ Radiodiagnosis, DNB, Shobha Diagnostic Centre, Dhiraj Apartments, Mumbai Malad East, India

*Corresponding Author: Rajesh C Kamble, Radiodiagnosis, Shobha Diagnostic Centre, Dhiraj Apartments, Mumbai Malad East, India.
Received: September 27, 2021

Published: October 26, 2021

(C) All rights are reserved by Rajesh C

Kamble., et al.

\begin{abstract}
Immunoglobulin G4 (IgG4)-related disease is an immune mediated disease causing fibro inflammatory response in the multiple organs of the human body It is a distinct clinical-pathologic entity more commonly seen in the elder populations and biochemically shows increased or elevated Igg4 levels.

Though IgG4RD most commonly seen in the elderly population, it can affect children and adolescents.

We report a case of an 18 year old male presenting with bilateral submandibular and parotid glands involvement in Igg 4 related disease diagnosed primarily on ultrasound to stress the importance of this diagnosis by pattern recognition on ultrasound imaging.

In the head and neck, the most common sites of involvement are the salivary glands, lacrimal glands, lymph nodes, and the gland. The pan involvement of bilateral parotid and submandibular glands and also lacrimal glands could be a clue to diagnose this disease process.
\end{abstract}

Keywords: IGG4 Disease; Submandibular Gland; Parotid Gland; Lacrimal Glands; Ultrasound

\section{Introduction}

Immunoglobulin G4 (IgG4)-related disease is an immune mediated disease causing fibro inflammatory response in the multiple organs of the human body It is a distinct clinical-pathologic entity more commonly seen in the elder populations and biochemically shows increased or elevated Igg4 levels. Most of these disease show excellent response to steroid therapy [1].

The head and neck involvement is commonly seen in this condition .The most commonly involved organs are salivary glands, lacrimal glands, orbits, thyroid gland, lymph nodes, sinonasal cavities, and pituitary stalk [1,2].
Though IgG4RD most commonly seen in the elderly population, it can affect children and adolescents. As the manifestations of this disease is multicentric, the radiology features often may be nonspecific making the diagnosis challenging and difficult in day to day radiology practice. Here, we report a case of an18 year old male presenting with bilateral submandibular and parotid glands involvement in Igg4 related disease diagnosed primarily on ultrasound to stress the importance of this diagnosis by pattern recognition on ultrasound imaging.

\section{Case History}

An 18 year old male patient presented to the surgeon with history of bilateral submandibular swellings. There were no consti- 
tutional symptoms. The patient gave history of gradually increasing bilateral submandibular swellings. There was a past history of surgery done for lymph nodes on the right side of the neck (proven Kikuchi's Fuji moto lymphadenopathy) 3 years back.

The surgeon after clinical examination made a preliminary diagnosis of recurrence of the same above mentioned disease process and asked for the CT/MRI neck for further evaluation.

The findings were reported as Multiple subcentimetre and centimeter sized bilateral submandibular, deep cervical and supraclavicular lymph nodes and bilateral bulky parotid glands with multiple small intraparotid lymph nodes consistent with previously diagnosed Kikuchi's lymphadenopathy.

The patient was put on symptomatic treatment but there was no resolution of the submandibular swellings after 2 weeks of treatment and hence referred to our centre for ultrasound evaluation.

\section{The USG findings revealed}

The thyroid lobes on the either side were enlarged .They were in homogenous and hypoechoic in echotexture. On Colour Doppler, there is increased vascularity seen within both lobes diffusely. The right hemi thyroid measured $3.7 \times 1.3 \times 2.1 \mathrm{~cm}$ in dimensions. The left hemi thyroid measured $3.5 \times 1.2 \times 1.4 \mathrm{~cm}$ in dimensions. No focal cystic and solid lesion noted in the thyroid gland. No evidence of calcification is noted.

The thyroid isthmus measured $0.59 \mathrm{~cm}$ in dimensions.

The bilateral parotid and submandibular glands were enlarged and in homogenous in appearance.

The visualized lacrimal glands appeared normal.

There were multiple hypo echoic lesions seen in the left submandibular space and left anterior triangle region.

They suggested enlarged lymph nodes. No necrosis seen. The largest of these lymph nodes measured $10 \times 10 \mathrm{~mm}$ and $15 \times 15$ $\mathrm{mm}$ in dimensions.

There were few enlarged reactive lymph nodes seen in the right anterior triangle, largest measuring $12 \times 12 \mathrm{~mm}$ in dimensions.

Visualized carotids and jugular vessels appear normal.

As the patients had non resolution of the symptoms and diffuse bilateral involvement of both parotids and submandibular glands, we raised a possibility of Igg4 related disease involvement in adolescent age group and requested for biochemical and histopathological evaluation.

The biochemical work up further strengthened our diagnosis as the serum Igg4 levels were markedly elevated. The levels were 7.8 $\mathrm{g} / \mathrm{L}$ (1.56-3.37 g/L).The ACE levels were normal.

The patient was subjected to USG guided biopsy of the left submandibular lymph nodal mass and the histopathology revealed a marked lymphoplasmacytic infiltration and fibrosis with IgG4+ plasma cells at histology which clinched the diagnosis we had proposed on ultrasound.

\section{Review of Literature and Discussion}

Chronic-sclerosing sialadenitis is an immune-mediated condition of the salivary glands that was first described in 1896.It is commonly referred as Kuttner tumour seen in elderly population showing isolated enlargement of the submandibular glands. The other causes of salivary gland enlargement are Sjögren syndrome and Mikulicz disease. Sjögren syndrome is an autoimmune disease primarily affecting salivary and lacrimal glands and commonly seen in female population. Mikulicz disease was initially described in the $19^{\text {th }}$ century to characterize idiopathic chronic enlargement of the submandibular, parotid, and lacrimal glands [1-3].

High IgG4 titers are seen in bronchiectasis, primary sclerosing cholangitis, vasculitis, and the newly diagnosed IgG4-related disease (IgG4RD) [1,4,5].

IgG4RD disease has evolved in last decade and hence it's a challenge to diagnose these diseases. Its multi organ involvement and atypical presentation with varied symptomatology can be a confusing clinical presentation and radiological interpretation.

The differential diagnosis involving pan - sialodenitis can be causes such as infection (commonest tuberculosis in India), lymphoma, Sjögren syndrome, sarcoidosis, and sialodocholithiasis $[1,2]$.

IgG4RD related diseases commonly seen in adult age group above 50 years and present commonly with symptoms involving the salivary and lacrimal glands, pancreas, and lymph nodes.

IgG4RD involvement in pediatric and adolescent group is less known entity and have been discussed mainly in case reports. In 
the pediatric population, pancreatic involvement was less common, whereas head and neck involvement continues to predominant [2].

IgG4RD is confirmed on histology by at least one of the following criteria - dense lymphoplasmacytic infiltrate, storiform-type fibrosis, or obliterative phlebitis $[1,4,5]$.

Serum IgG4 is elevated in majority of the cases [1].

The combination of the patient clinical presentation, elevated serum IgG4 titers, imaging studies and histopathological findings clinches the diagnosis of IgG4RD $[1,4,5]$.

Radiological studies often reveal nonspecific findings such as diffuse organ enlargement, homogeneous contrast enhancement, and lymphadenopathy of $1-3 \mathrm{~cm}$ in diameter. CT and MRI are preferred modalities [3].

Although the radiologic findings associated with IgG4-related disease may be nonspecific, it is important for radiologists to be familiar with this disease process to raise suspicion for this disease on imaging. In the head and neck, the most common sites of involvement are the salivary glands, lacrimal glands, lymph nodes, and the gland. The pan involvement of bilateral parotid and submandibular glands could be a clue to diagnose this disease process [1].

Radiologists who practice Ultrasound, CT and MRI can play a great role by raising the suspicion of this disease process.

Corticosteroids are accepted as first-line therapy for IgG4RD, including pediatric population with good recovery rate $[1,4,5]$. The disease can underdo remission and relapse and hence these patients need to follow up for better outcome.

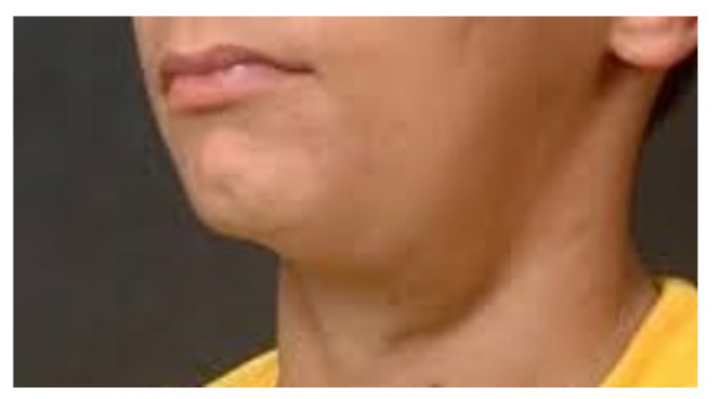

Image 1: Clinical image - enlarged left submandibular gland.

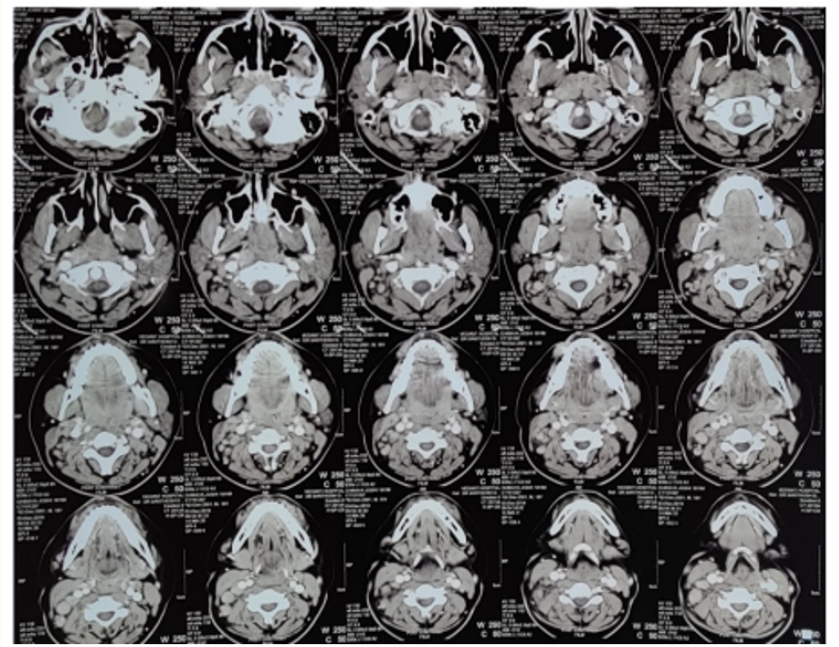

Image 2: CT scan images of neck.

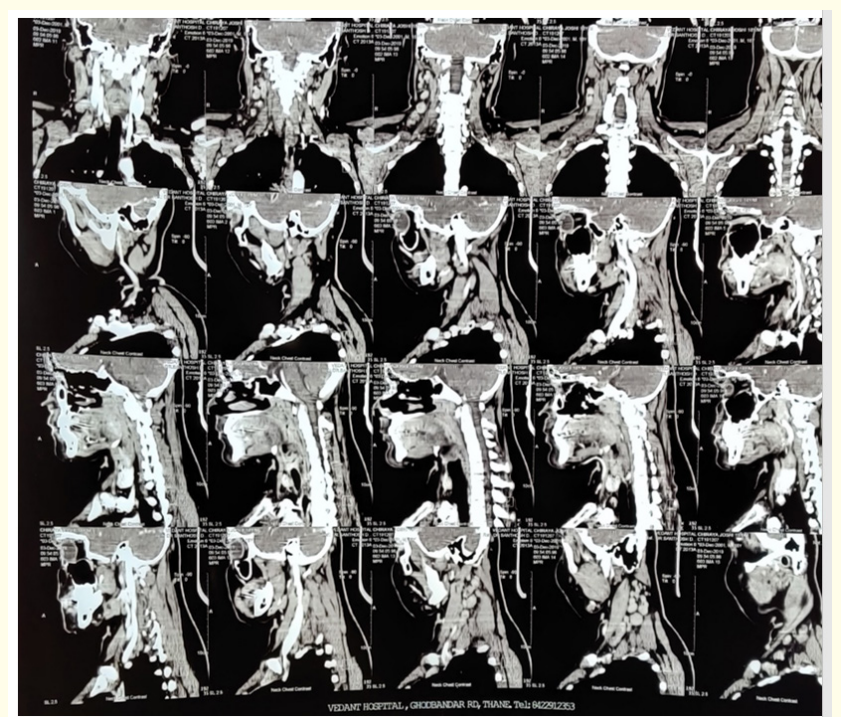

Image 3: Axial/coronal and MPR CT scan images of neck showing enlarged submandibular glands and cervical adenopathy. 

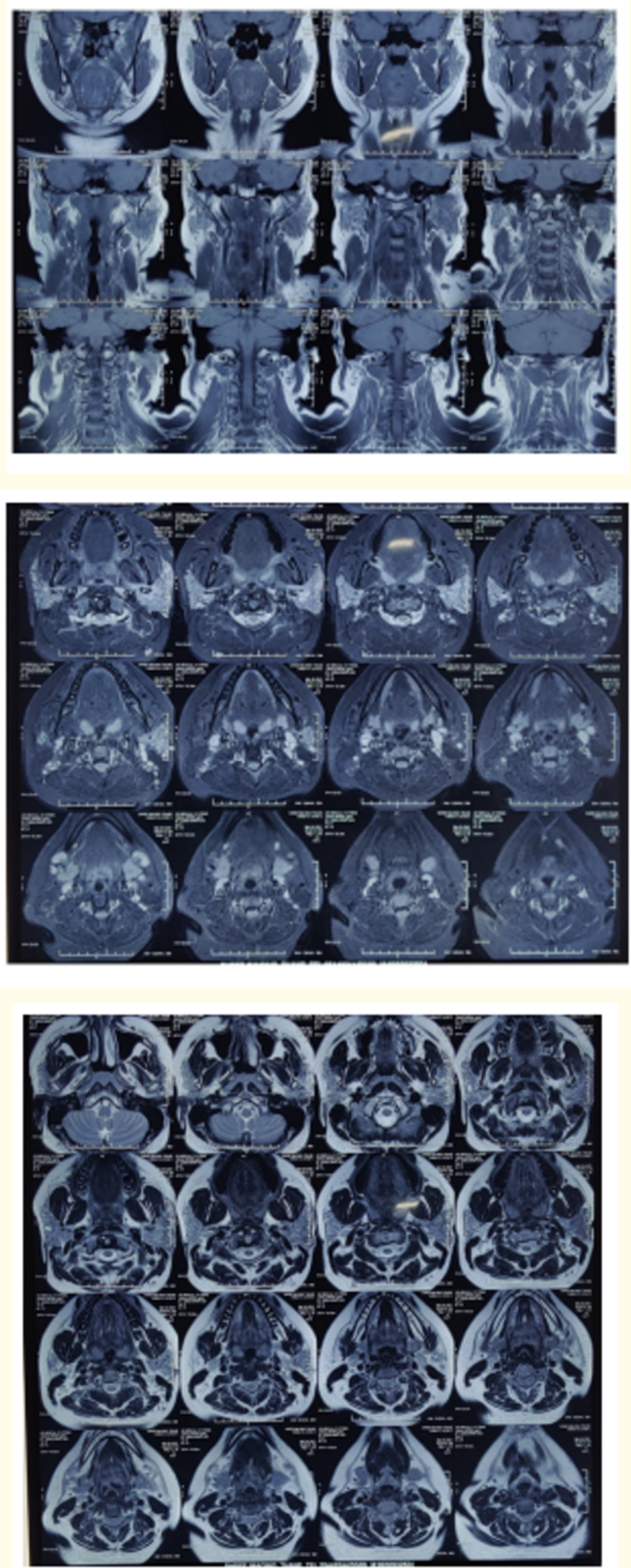

Image 4: MRI images showing enlarged cervical lymph nodes and enlarged parotid and submandibular glands.

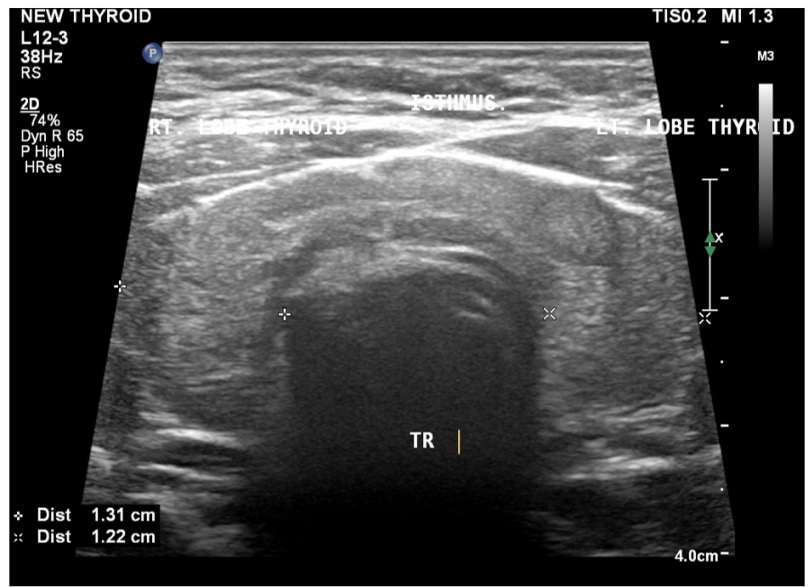

Image 5: Ultrasound images. B mode images of both thyroid glands and isthmus. Images show normal bilateral thyroid glands and isthmus.
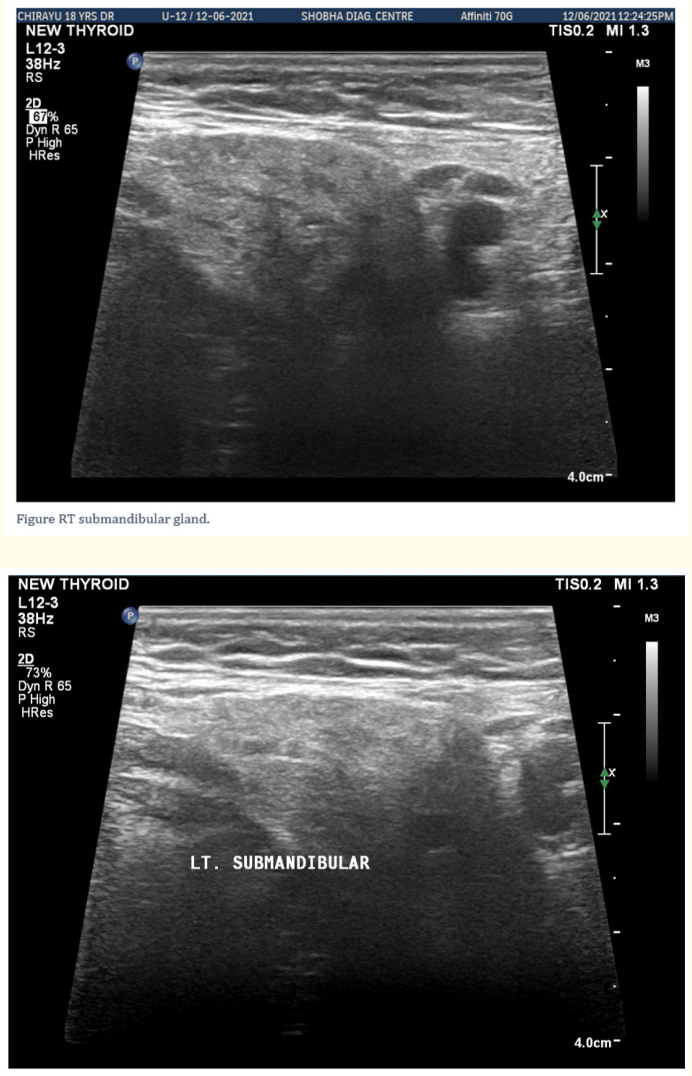

Image 6: B mode images of both submandibular glands showing bilateral in homogenous enlarged glands. 


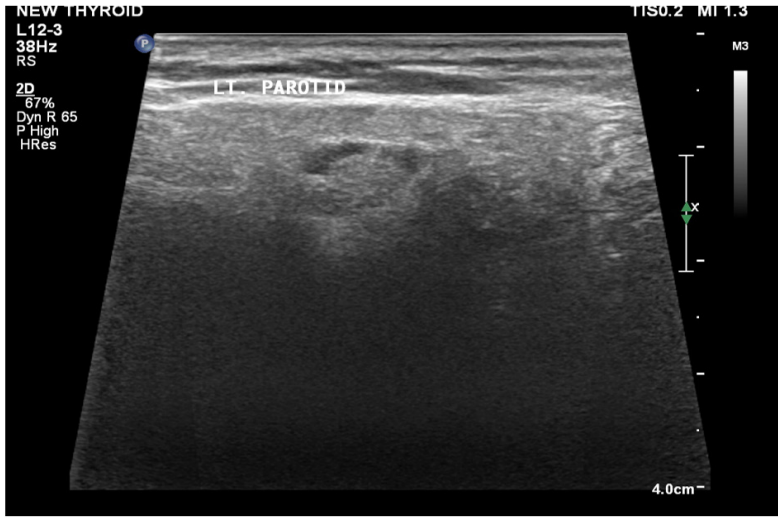

Image 7: B mode images of bilateral parotid glands showing bilateral in homogenous parotid glands.

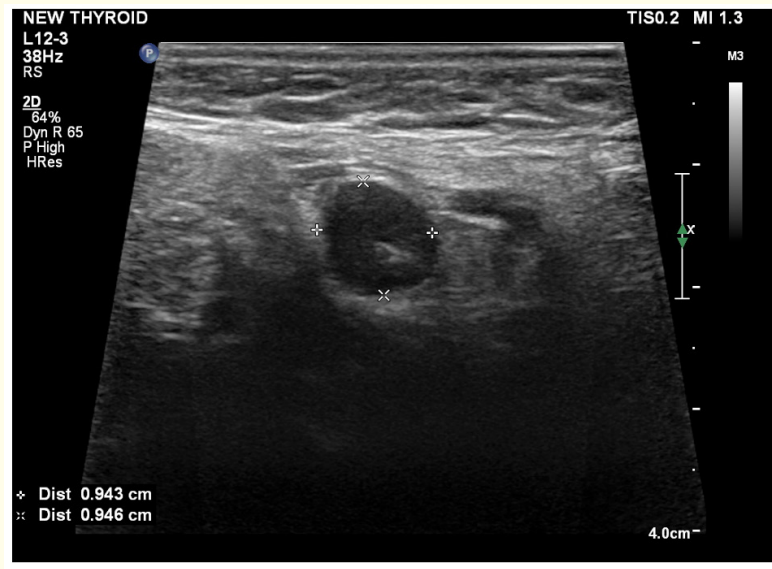

Images 8: USG B mode images showing enlarged cervical lymph nodes.

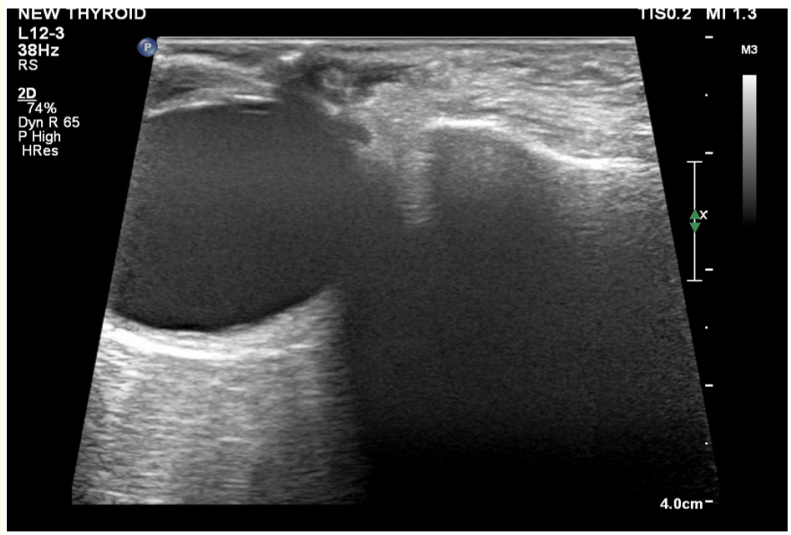

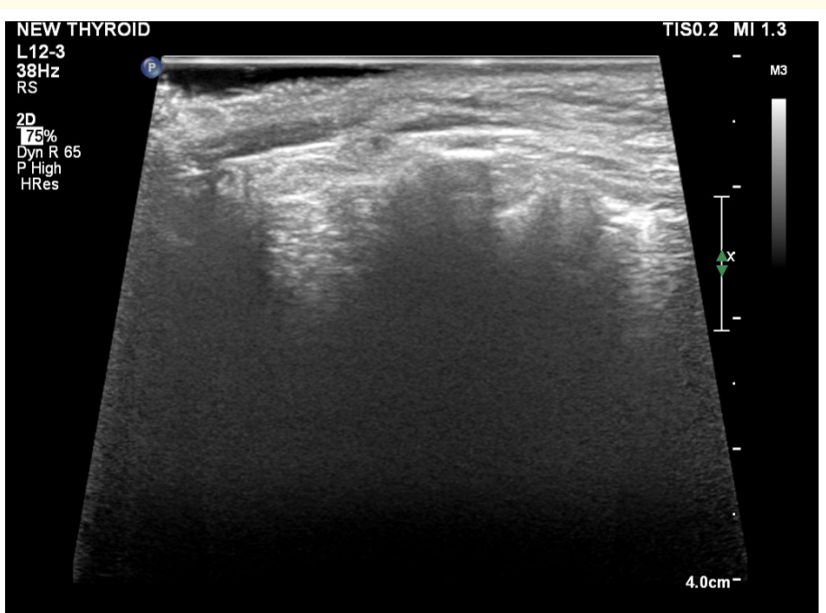

Image 9: B mode images of normal bilateral lacrimal glands.

\section{Conclusion}

IgG4RD is a systemic disease that can affect many different organs. Although the imaging features of IgG4RD can be nonspecific, imaging specialist can play a primary role in suspecting the disease process and reporting it. Ultrasound due to its inherent spatial resolution and tissue perception can help confidently raise suspicion of this disease process when there is bilateral involvement of the salivary and lacrimal glands, helping the clinician to investigate further and biochemically and histologically achieve the diagnosis. Pattern recognition with bilateral submandibular, parotid and lacrimal involvement with inhomogeneous echotexture could be a strong clue and pointer to this disease process.

\section{Bibliography}

1. Ilaria Puxeddu., et al. "Salivary Gland Pathology in IgG4-Related Disease: A Comprehensive Review". Journal of Immunology Research 2018 (2018): 6936727.

2. Renato Cesar Ferreira da Silva., et al. "IgG4-related disease in an adolescent with radiologic-pathologic correlation". Radiology Case Reports 12.1 (2017): 196-199.

3. Laurens J De Cocker., et al. "IgG4-related systemic disease affecting the parotid and submandibular glands: magnetic resonance imaging features of IgG4-related chronic sclerosing sialadenitis and concomitant lymphadenitis". Clinical Imaging 38.2 (2014): 195-198. 
4. Vikram Deshpande., et al. "Consensus statement on the pathology of IgG4-related disease". Modern Pathology 25 (2012): 1181-1192.

5. Torsten Witte and Hendrik Schulze-Koops. "IgG4-related sialadenitis: IgG4 is helpful, but biopsies are still crucial". Arthritis Research and Therapy 17 (2015): 368.

Volume 3 Issue 11 November 2021

(c) All rights are reserved by Rajesh C Kamble., et al. 